TRANSACTIONS OF THE

AMERICAN MATHEMATICAL SOCIETY

Volume 352, Number 11, Pages 5279-5292

S 0002-9947(00)02600-3

Article electronically published on July 12, 2000

\title{
PATH STABILITY AND NONLINEAR WEAK ERGODIC THEOREMS
}

\author{
YONG-ZHUO CHEN
}

\begin{abstract}
Let $\left\{f_{n}\right\}$ be a sequence of nonlinear operators. We discuss the asymptotic properties of their inhomogeneous iterates $f_{n} \circ f_{n-1} \circ \cdots \circ f_{1}$ in metric spaces, then apply the results to the ordered Banach spaces through projective metrics. Theorems on path stability and nonlinear weak ergodicity are obtained in this paper.
\end{abstract}

\section{IntRoduction}

The change of a phenomenon at discrete points of time can be modeled by a discrete dynamical system defined by an operator $f$. If the system itself changes over time, then we have to consider a sequence of operators $\left\{f_{n}\right\}$ and the inhomogeneous iterations $f_{n} \circ f_{n-1} \circ \cdots \circ f_{1}$, which can be considered as a generalization of inhomogeneous products of matrices (Seneta [16]). In this paper, we are interested in the cases where $f_{n}$ 's are nonlinear and certain asymptotic properties of their inhomogeneous iterates are discussed.

Let $x, y$ be any two points in a space. If

$$
\lim _{n \rightarrow \infty} d\left(f_{n} \circ f_{n-1} \circ \cdots \circ f_{1}(x), f_{n} \circ f_{n-1} \circ \cdots \circ f_{1}(y)\right)=0
$$

or

$$
\lim _{n \rightarrow \infty}\left\|f_{n} \circ f_{n-1} \circ \cdots \circ f_{1}(x)-f_{n} \circ f_{n-1} \circ \cdots \circ f_{1}(y)\right\|=0,
$$

where $d(\cdot, \cdot)$ is some metric and $\|\cdot\|$ is some norm, then we say that $f_{n}$ is path stable (Krause $[12,13])$. Section 2 will be devoted to path stabilities in metric spaces. In Section 3, we apply the results in Section 2 to the path stabilities in ordered Banach spaces by means of Thompson's metric.

Let $T_{n}(x)=\frac{f_{n}(x)}{\left\|f_{n}(x)\right\|}$ be the rescaled operator. If

$$
\lim _{n \rightarrow \infty}\left\|T_{n} \circ T_{n-1} \circ \cdots \circ T_{1}(x)-T_{n} \circ T_{n-1} \circ \cdots \circ T_{1}(y)\right\|=0,
$$

then we say that $T_{n}$ is weakly ergodic (Fujimoto and Krause [6], Inaba [8], Nussbaum [15]). The weak ergodic theorems are extensively studied and applied in population biology literature. In Section 4, we apply the results in Section 2 and the Hilbert metric to prove several nonlinear weak ergodic theorems in the ordered

Received by the editors June 30, 1998 and, in revised form, June 1, 1999.

2000 Mathematics Subject Classification. Primary 47H07, 47H09; Secondary 47H10.

Key words and phrases. Hilbert metric, inhomogeneous iterates, metric space, monotone operator, ordered Banach space, Thompson's metric. 
Banach spaces. As pointed out in [4] Remark 4.2] (cf. [3, Corollary 3.2]), our operators in Section 3 and Section 4 are related to the ascending operators (Fujimoto and Krause [6], Krause [13]).

In Section 5, we explore the application of our theorems to the ranking of $n$ teams in a competition game.

\section{Path Stability in metric Space}

In this section, $(X, d)$ stands for a complete metric space. A sequence of operators $T_{n}: X \rightarrow X, n=1,2, \cdots$, is said to be asymptotically generalized contractive (cf. Fujimoto and Krause [6], also M. A. Krasnosel'skiı̌ and P. P. Zabreǐko [11, p. 206]) if for any $[a, b] \subset(0, \infty)$ and $\epsilon>0$, there exists $L(a, b) \in(0,1)$ and $N=N(\epsilon, a, b)>0$ such that

$$
d\left(T_{n}(x), T_{n}(y)\right) \leq L(a, b) d(x, y)+\epsilon \quad \text { if } d(x, y) \in[a, b],
$$

and $d\left(T_{n}(x), T_{n}(y)\right) \leq d(x, y)+\epsilon$ otherwise, where $n>N$ and $x, y \in X$.

First we prove a lemma which is a strengthening of [4 Lemma 2.1] (also see Jachymski [9, Lemma 1]).

Lemma 2.1. Let $\left\{u_{n}\right\}$ be a sequence of positive numbers. Suppose for any $[a, b] \subset$ $(0, \infty)$, there exists $L(a, b) \in(0,1)$, and for a given $\epsilon>0$, we have $N=N(\epsilon, a, b)>$ 0 such that

$$
u_{n+1} \leq L(a, b) u_{n}+\epsilon \quad \text { if } u_{n} \in[a, b],
$$

and $u_{n+1} \leq u_{n}+\epsilon$ otherwise, where $n \geq N$. Then either $\lim _{n \rightarrow \infty} u_{n}=0$ or $\left\{u_{n}\right\}$ is unbounded.

Proof. Suppose that $b=\sup \left\{u_{n}\right\}<\infty$. Assume that $\lim _{n \rightarrow \infty} u_{n}=0$ is not true. Then we can claim that there exists $m>0$ such that $u_{n} \geq m$ for all but finitely many $n$. For if not, there exists $\delta \in(0, b)$ such that both $\left\{n: u_{n} \leq \delta\right\}$ and $\left\{n: u_{n}>\delta\right\}$ are infinite sets. Let

$$
n_{1}=\min \left\{n: u_{n} \leq \delta \text { and } u_{n+1}>\delta\right\} .
$$

Using induction, we have

$$
n_{k+1}=\min \left\{n>n_{k}: u_{n} \leq \delta \text { and } u_{n+1}>\delta\right\} .
$$

For this selected $\left\{u_{n_{k}}\right\}$, we must have $\liminf _{k \rightarrow \infty} u_{n_{k}} \geq \frac{\delta}{3}$. Since otherwise, there is a subsequence of $\left\{u_{n_{k}}\right\}$, we still denote it by $\left\{u_{n_{k}}\right\}$ for simplicity, such that $u_{n_{k}}<\frac{\delta}{2}$ for all $k$. For $\epsilon_{1}=\frac{\delta}{4}$, there exists $N_{1}=N_{1}\left(\epsilon_{1}, \frac{\delta}{2}, b\right)>0$ such that

$$
u_{n_{k}+1} \leq u_{n_{k}}+\epsilon_{1}
$$

for all $n_{k} \geq N_{1}$. This implies that $\delta \leq \frac{\delta}{2}+\frac{\delta}{4}$, which is a contradiction. Hence, without loss of generality, we can assume that $u_{n_{k}} \geq \frac{\delta}{4}$ for all $n_{k}>N$. Again by (2), for any $\epsilon_{2}>0$, there exists $N_{2}=N_{2}\left(\epsilon_{2}, \frac{\delta}{4}, b\right)>0$ such that

$$
u_{n_{k}+1} \leq L\left(\frac{\delta}{4}, b\right) u_{n_{k}}+\epsilon_{2}
$$

for all $n_{k}>N_{2}$. This leads to $\delta \leq L\left(\frac{\delta}{4}, b\right) \delta+\epsilon_{2}$ due to the definition of $n_{k}$. It is impossible since $L\left(\frac{\delta}{4}, b\right)<1$ and $\epsilon_{2}>0$ can be arbitrary. The claim is proved. Therefore in the following we can assume $m=\inf \left\{u_{n}\right\}$. 
Given $\epsilon>0$, the contraction mapping $f(x)=L(m, b) x+\epsilon$ has a unique fixed point $\frac{\epsilon}{1-L(m, b)}$. There exists $N=N(\epsilon, m, b)>0$ such that

$$
u_{n+1} \leq L(m, b) u_{n}+\epsilon=f\left(u_{n}\right)
$$

for all $n \geq N$. So

$$
\begin{aligned}
u_{n+2} & \leq L(m, b) u_{n+1}+\epsilon=f\left(u_{n+1}\right) \\
& \leq f^{2}\left(u_{n}\right) \quad(\text { by }(3) \text { and the monotonity of } f) .
\end{aligned}
$$

By induction, we have $u_{n+p} \leq f^{p}\left(u_{n}\right), p \geq 1$. Using the facts that $\inf \left\{u_{n}\right\}=m$ and $\lim _{p \rightarrow \infty} f^{p}\left(u_{n}\right)=\frac{\epsilon}{1-L(m, b)}$, we have $m \leq \frac{\epsilon}{1-L(m, b)}$. It is impossible since $\epsilon>0$ can be arbitrary.

Remark 1. In [4, Lemma 2.1], $\epsilon_{n} \rightarrow 0$ uniformly for all $[a, b] \subset(0, \infty)$, whereas here we require $N(\epsilon, a, b)$ depending on $a$ and $b$.

Remark 2. To complement condition (2), we assume that $u_{n+1} \leq u_{n}+\epsilon$, otherwise. This is necessary. For example, consider the bounded sequence

$$
\left\{1, \frac{1}{2}, 1, \frac{1}{3}, 1, \frac{1}{4}, 1, \frac{1}{5}, \cdots\right\} \text {. }
$$

It satisfies (2) since we can let $L(a, b)=\frac{1}{2}$ for all $[a, b] \subset(0, \infty)$. Then for any given $[a, b] \subset(0, \infty)$ and $\epsilon>0$, we can choose $N(\epsilon, a, b)>\frac{1}{a}$ to satisfy (2). However, the sequence is not convergent.

Theorem 2.2. Let $\left\{T_{n}\right\}$ be a sequence of asymptotically generalized contractions on $X$. Let $x_{1}, y_{1} \in X$, and define

$$
x_{n+1}=T_{n}\left(x_{n}\right) \text { and } y_{n+1}=T_{n}\left(y_{n}\right) .
$$

Then either $\lim _{n \rightarrow \infty} d\left(x_{n}, y_{n}\right)=0$ or $\left\{d\left(x_{n}, y_{n}\right): n \geq 1\right\}$ is unbounded.

Proof. Letting $u_{n}=d\left(x_{n}, y_{n}\right)$ and an application of Lemma 2.1 yield the theorem.

We would like to remark that (1) does not imply all $T_{n}$ 's are continuous. If the condition (1) is strengthened, then we can have the following

Corollary 2.3. Let $T_{n}: X \rightarrow X, n=1,2, \cdots$. Suppose for any $0<a<b<\infty$, there exists $L(a, b) \in(0,1)$ such that

$$
d\left(T_{n}(x), T_{n}(y)\right) \leq L(a, b) d(x, y)
$$

for all $n$, where $x, y \in X$ and $a \leq d(x, y) \leq b$. Let $x_{1}, y_{1} \in X$, and define

$$
x_{n+1}=T_{n}\left(x_{n}\right) \text { and } y_{n+1}=T_{n}\left(y_{n}\right) .
$$

Then $\lim _{n \rightarrow \infty} d\left(x_{n}, y_{n}\right)=0$.

Proof. Condition (4) implies that $d\left(x_{n}, y_{n}\right) \leq d\left(x_{1}, y_{1}\right)$ for all $n$, i.e., $\left\{d\left(x_{n}, y_{n}\right)\right\}$ is bounded. The conclusion follows from Theorem 2.2 .

A sequence of operators $T_{n}: X \rightarrow X, n=1,2, \cdots$, is said to be asymptotically large contractive (cf. Burton [2]) if for any $a>0$ and $\epsilon>0$, there exists $L(a) \in$ $(0,1)$ and $N=N(\epsilon, a)>0$ such that

$$
d\left(T_{n}(x), T_{n}(y)\right) \leq L(a) d(x, y)+\epsilon \quad \text { if } u_{n} \geq a,
$$

and $d\left(T_{n} x, T_{n} y\right) \leq d(x, y)+\epsilon$, otherwise, where $n>N$ and $x, y \in X$. 
To justify the introduction of the above definition, we give an example which is asymptotically generalized but not large contractive. This example is a modification of the one given by Boyd and Wong (4, Remark 3]).

Example. Let $X=[0,1] \cup\{2,3,4, \cdots\}$, and

$$
d(x, y)= \begin{cases}|x-y| & \text { if } x, y \in[0,1], \\ x+y & \text { if at least one of } x, y \bar{\in}[0,1] .\end{cases}
$$

$(X, d)$ is a complete metric space ([1, Remark 3]). Define

$$
T_{n}= \begin{cases}x-\frac{1}{2} x^{2}+\frac{1}{n} x, & x \in[0,1], \\ x-1+\frac{1}{n} x, & x=2,3, \cdots .\end{cases}
$$

Now, for $x, y \in[0,1]$ with $x-y=t>0$,

$$
\begin{aligned}
d\left(T_{n} x, T_{n} y\right) & =x-\frac{1}{2} x^{2}+\frac{1}{n} x-y+\frac{1}{2} y^{2}-\frac{1}{n} y \\
& =(x-y)\left(1-\frac{1}{2}(x+y)\right)+\frac{1}{n}(x-y) \\
& \leq t\left(1-\frac{1}{2} t\right)+\frac{1}{n} t .
\end{aligned}
$$

On the other hand, for $x \in\{2,3, \cdots\}$ with $x>y$,

$$
\begin{aligned}
d\left(T_{n} x, T_{n} y\right) & =T_{n} x+T_{n} y \\
& <x-1+\frac{1}{n} x+y+\frac{1}{n} y \\
& =d(x, y)-1+\frac{1}{n} d(x, y) .
\end{aligned}
$$

Define

$$
\psi(t)= \begin{cases}t-\frac{1}{2} t^{2}, & t \in[0,1] \\ t-1, & t>1\end{cases}
$$

Then

$$
d\left(T_{n} x, T_{n} y\right) \leq \psi(d(x, y))+\frac{1}{n} d(x, y) .
$$

For any $[a, b] \subset(0, \infty)$, let

$$
L(a, b)=\max \left\{\frac{\psi(d(x, y))}{d(x, y)}: a \leq d(x, y) \leq b\right\},
$$

and for any $\epsilon>0$, let $N=N(\epsilon, a, b)=\left[\frac{b}{\epsilon}\right]$. It follows that for all $n>N, x, y \in X$ with $d(x, y) \in[a, b]$, we have

$$
d\left(T_{n} x, T_{n} y\right) \leq L(a, b) d(x, y)+\epsilon .
$$

Hence $\left\{T_{n}\right\}$ is asymptotically generalized but not large contractive since $\lim _{n \rightarrow \infty} \frac{\psi(d(n, k))}{d(n, k)}=1$ for any fixed positive integer $k$.

We need the following lemma which is a strengthening of [4, Lemma 3.1].

Lemma 2.4. Let $\left\{u_{n}\right\}$ be a sequence of positive numbers. Suppose for any a $>0$ and $\epsilon>0$, there exists $L(a) \in(0,1)$ and $N=N(\epsilon, a)>0$ such that

$$
u_{n+1} \leq L(a) u_{n}+\epsilon \quad \text { if } u_{n} \geq a,
$$

and $u_{n+1} \leq u_{n}+\epsilon$, otherwise, where $n \geq N$. Then $\lim _{n \rightarrow \infty} u_{n}=0$. 
Proof. By Lemma 2.1, we only need to prove that $\left\{u_{n}\right\}$ is bounded.

Assume that $\left\{u_{n}\right\}$ is unbounded. Then for each positive integer $k$, we put $n_{k}=\inf \left\{n: u_{n} \geq k\right\}$. There are two possibilities for $\left\{u_{n_{k}-1}\right\}$.

Case I. $\left\{u_{n_{k}-1}\right\}$ is bounded. This will lead to a contradiction in view of the assumptions on $\left\{u_{n}\right\}$.

Case II. $\left\{u_{n_{k}-1}\right\}$ is unbounded. Then there exists a monotone increasing subsequence of $\left\{u_{n_{k}-1}\right\}$, which diverges to $\infty$ and is still denoted by $\left\{u_{n_{k}-1}\right\}$ for simplicity. Without loss of generality, we assume $u_{n_{k}-1} \geq 1$. For any given $\epsilon>0$, there exists $L(1) \in(0,1)$ and $N_{1}=N_{1}(\epsilon, 1)>0$ such that

$$
u_{n_{k}} \leq L(1) u_{n_{k}-1}+\epsilon
$$

for all $n_{k}-1>N_{1}$. The selection of $n_{k}$ implies $k \leq L(1) k+\epsilon$. From this we have $(1-L(1)) k \leq \epsilon$, which is impossible since $k \rightarrow \infty$.

Theorem 2.5. Let $\left\{T_{n}\right\}$ be a sequence of asymptotically large contractions on $X$. Let $x_{1}, y_{1} \in X$, and define

$$
x_{n+1}=T_{n}\left(x_{n}\right) \text { and } y_{n+1}=T_{n}\left(y_{n}\right) .
$$

Then $\lim _{n \rightarrow \infty} d\left(x_{n}, y_{n}\right)=0$.

Proof. Letting $u_{n}=d\left(x_{n}, y_{n}\right)$ and an application of Lemma 2.4 yield the theorem.

As in [6], we can consider the sequence of lumped operators. For a given $l>1$ and a given sequence $\left\{T_{n}\right\}$ of operators on $X$, the sequence of lumped operators $\left\{F_{m}\right\}$ is defined by $F_{m}=T_{m+l-1} \circ T_{m+l-2} \circ \cdots \circ T_{m+1} \circ T_{m}$. An operator $T$ on $X$ is called a Lipschitz operator if $d(T x, T y) \leq r d(x, y)$ for all $x, y \in X$, where $r>0$ is the Lipschitz constant.

Theorem 2.6. Let $\left\{T_{n}\right\}$ be a sequence of Lipschitz operators on $X$ with the same Lipschitz constant $r$. Suppose that for some $l>1$, the sequence of lumped operators $\left\{F_{m}\right\}$ is asymptotically generalized contractive. Let $x_{1}, y_{1} \in X$, and define

$$
x_{n+1}=T_{n}\left(x_{n}\right) \text { and } y_{n+1}=T_{n}\left(y_{n}\right) .
$$

Then either $\lim _{n \rightarrow \infty} d\left(x_{n}, y_{n}\right)=0$ or $\left\{d\left(x_{n}, y_{n}\right): n \geq 1\right\}$ is unbounded.

Proof. Suppose that $\left\{d\left(x_{n}, y_{n}\right)\right\}$ is bounded. Let $S_{m}=F_{(m-1) l+1}$ and $\bar{x}_{m+1}=$ $S_{m}\left(\bar{x}_{m}\right), \bar{y}_{m+1}=S_{m}\left(\bar{y}_{m}\right)$, where $\bar{x}_{1}=x_{1}, \bar{y}_{1}=y_{1}$. It is clear that $\left\{d\left(\bar{x}_{m}, \bar{y}_{m}\right)\right\}$ is bounded by assumption. Note that $\bar{x}_{m+1}=x_{m l+1}, \bar{y}_{m+1}=y_{m l+1}$. Let $n>0$ be given. Then there exist nonnegative integers $m(n)$ and $i$ such that $n=m(n) l+i$ with $0 \leq i<l$. We have

$$
\begin{aligned}
d\left(x_{n+1}, y_{n+1}\right) & \leq r^{i} d\left(x_{m(n) l+1}, y_{m(n) l+1}\right) \\
& =r^{i} d\left(\bar{x}_{m(n)+1}, \bar{y}_{m(n)+1}\right) \\
& \leq \max \left\{1, r^{l}\right\} d\left(\bar{x}_{m(n)+1}, \bar{y}_{m(n)+1}\right) .
\end{aligned}
$$

By Theorem 2.2, $\lim _{n \rightarrow \infty} d\left(\bar{x}_{m(n)+1}, \bar{y}_{m(n)+1}\right)=0$. Hence $d\left(x_{n+1}, y_{n+1}\right) \rightarrow 0$ as $n \rightarrow \infty$.

Corollary 2.7. Let $\left\{T_{n}\right\}$ be a sequence of Lipschitz operators on $X$ with the same Lipschitz constant $r$. Suppose for some $l>1$, the sequence of lumped operators 
$\left\{F_{m}\right\}$ satisfy the following: For any $0<a<b<\infty$, there exists $L(a, b) \in(0,1)$ such that

$$
d\left(F_{m}(x), F_{m}(y)\right) \leq L(a, b) d(x, y)
$$

for all $m$, where $x, y \in X$ and $a \leq d(x, y) \leq b$. Let $x_{1}, y_{1} \in X$, and define

$$
x_{n+1}=T_{n}\left(x_{n}\right) \text { and } y_{n+1}=T_{n}\left(y_{n}\right) \text {. }
$$

Then $\lim _{n \rightarrow \infty} d\left(x_{n}, y_{n}\right)=0$.

Proof. We only need to point out that

$$
d\left(x_{n}, y_{n}\right) \leq \max \left\{1, r^{l}\right\} d\left(x_{1}, y_{1}\right)
$$

for all $n$, i.e., $\left\{d\left(x_{n}, y_{n}\right)\right\}$ is bounded. Then the conclusion follows from Theorem 2.6 .

Similarly, we can prove the following theorem.

Theorem 2.8. Let $\left\{T_{n}\right\}$ be a sequence of Lipschitz operators on $X$ with the same Lipschitz constant $r$. Suppose that for some $l>1$, the sequence of lumped operators $\left\{F_{m}\right\}$ is asymptotically large contractive. Let $x_{1}, y_{1} \in X$, and define

$$
x_{n+1}=T_{n}\left(x_{n}\right) \text { and } y_{n+1}=T_{n}\left(y_{n}\right) .
$$

Then $\lim _{n \rightarrow \infty} d\left(x_{n}, y_{n}\right)=0$.

\section{Path Stability in ORdered BANACH SPACES}

In the rest of this paper, $(B,\|\cdot\|)$ stands for a real Banach space which is partially ordered by a closed convex cone $P$. Suppose the norm is monotone, i.e., $x \leq y$ implies that $\|x\| \leq\|y\|$. Note that $P$ is normal iff $B$ has an equivalent norm

which is monotone. $\stackrel{\circ}{P}$ denotes the interior of $P$, and $P$ is solid if $\stackrel{\circ}{P}$ is not empty. $x, y \in P-0$ are called comparable (Nussbaum [14]) if there exist $\lambda, \mu>0$ such that $\lambda x \leq y \leq \mu x$. This partitions $P-0$ into disjoint equivalent classes which are called components of $P$. An operator $f: D \subset B \rightarrow B$ is monotone if $x \leq y$ implies $f(x) \leq f(y)$, where $x, y \in D$.

Let $C$ be a component of $P$. For $x, y \in C$, let $M(x / y)=\inf \{\lambda: x \leq \lambda y\}$. Thompson's metric is defined by

$$
\bar{d}(x, y)=\ln \{\max [M(x / y), M(y / x)]\},
$$

and $(C, \bar{d})$ is a complete metric space (see Thompson [17]).

We need the following lemma.

Lemma 3.1. Let $\left\{x_{n}\right\}$ and $\left\{y_{n}\right\}$ be two sequences in $B$. If $\sup \left\{\left\|x_{n}\right\|\right\}<\infty$ and $\lim _{n \rightarrow \infty} \bar{d}\left(x_{n}, y_{n}\right)=0$, then $\lim _{n \rightarrow \infty}\left\|x_{n}-y_{n}\right\|=0$.

Proof. $\lim _{n \rightarrow \infty} \bar{d}\left(x_{n}, y_{n}\right)=0$ implies

$$
\lim _{n \rightarrow \infty} \max \left[M\left(x_{n} / y_{n}\right), M\left(y_{n} / x_{n}\right)\right]=1 .
$$

Then for any $\epsilon>0$, there exists $N>0$ such that

$$
M\left(x_{n} / y_{n}\right) \leq 1+\epsilon \text { and } M\left(y_{n} / x_{n}\right) \leq 1+\epsilon
$$

for all $n \geq N$. Hence for $n \geq N$, we have

$$
\frac{1}{1+\epsilon} x_{n} \leq y_{n} \leq(1+\epsilon) x_{n} \text {. }
$$


Thus

$$
-\frac{\epsilon}{1+\epsilon} x_{n} \leq y_{n}-x_{n} \leq \epsilon x_{n}
$$

Since the norm $\|\cdot\|$ is monotone,

$$
\begin{aligned}
\left\|y_{n}-x_{n}\right\| & \leq \max \left\{\frac{\epsilon}{1+\epsilon}, \epsilon\right\}\left\|x_{n}\right\| \\
& \leq \epsilon b, \quad n \geq N .
\end{aligned}
$$

Therefore $\lim _{n \rightarrow \infty}\left\|x_{n}-y_{n}\right\|=0$.

Theorem 3.2. Let $f_{n}: C \rightarrow C, n \geq 1$, be a sequence of monotone operators. Suppose for each $\left[l_{1}, l_{2}\right] \subset(0,1)$, there exists $\alpha_{n}\left(l_{1}, l_{2}\right)>0$ with $\lim \sup _{n \rightarrow \infty} \alpha_{n}\left(l_{1}, l_{2}\right)$ $<1$ such that

$$
y \geq t x \quad \text { implies } \quad f_{n}(y) \geq t^{\alpha_{n}\left(l_{1}, l_{2}\right)} f_{n}(x) \quad \text { if } t \in\left[l_{1}, l_{2}\right]
$$

and $f_{n}(y) \geq t f_{n}(x)$, otherwise, where $x, y \in C$. If there exists an $x_{0} \in C$ such that $\sup \left\{\left\|x_{n}\right\|\right\}<\infty$, where $x_{n}=f_{n}\left(x_{n-1}\right), n=1,2, \cdots$, then for any $y_{0} \in C$ and $y_{n}=f_{n}\left(y_{n-1}\right)$, we have the following trichotomy. Either

(i) $\left\{M\left(x_{n} / y_{n}\right)\right\}$ is unbounded; or

(ii) $\left\{M\left(y_{n} / x_{n}\right)\right\}$ is unbounded; or

(iii) $\lim _{n \rightarrow \infty}\left\|x_{n}-y_{n}\right\|=0$.

Proof. For $x, y \in C$ with $\bar{d}(x, y) \in[a, b] \subset(0, \infty)$. Without loss of generality, assume $M(x / y) \geq M(y / x)$. Then $e^{a} \leq M(x / y) \leq e^{b}$. Since $y \geq\left[\frac{1}{M(x / y)}\right] x$, we have

$$
f_{n}(y) \geq\left[\frac{1}{M(x / y)}\right]^{\alpha_{n}\left(e^{-b}, e^{-a}\right)} f_{n}(x) \quad \text { by }(8) .
$$

On the other hand, $x \geq\left[\frac{1}{M(y / x)}\right] y \geq\left[\frac{1}{M(x / y)}\right] y$. Again, (8) implies

$$
f_{n}(x) \geq\left[\frac{1}{M(x / y)}\right]^{\alpha_{n}\left(e^{-b}, e^{-a}\right)} f_{n}(y) .
$$

It follows that

$$
M\left(f_{n}(x) / f_{n}(y)\right) \leq M(x / y)^{\alpha_{n}\left(e^{-b}, e^{-a}\right)}
$$

and

$$
M\left(f_{n}(y) / f_{n}(x)\right) \leq M(x / y)^{\alpha_{n}\left(e^{-b}, e^{-a}\right)} .
$$

Hence

$$
\bar{d}\left(f_{n}(x), f_{n}(x)\right) \leq \alpha_{n}\left(e^{-b}, e^{-a}\right) \bar{d}(x, y) .
$$

Let $L(a, b)=\limsup _{n \rightarrow \infty} \alpha_{n}\left(e^{-b}, e^{-a}\right)<1$. Then for any given $\epsilon>0$, there exists $N>0$ such that

$$
\alpha_{n}\left(e^{-b}, e^{-a}\right) \leq L(a, b)+\frac{\epsilon}{b}
$$

for all $n>N$. By (9), we have

$$
\begin{aligned}
\bar{d}\left(f_{n}(x), f_{n}(y)\right) & \leq\left(L(a, b)+\frac{\epsilon}{b}\right) \bar{d}(x, y) \\
& \leq L(a, b) \bar{d}(x, y)+\epsilon
\end{aligned}
$$

for all $n>N$. 
For $x, y \in C$ with $\bar{d}(x, y) \bar{\epsilon}[a, b]$, by a similar argument, we have $\bar{d}\left(f_{n}(x), f_{n}(y)\right)$ $\leq \bar{d}(x, y)$ due to $f_{n}(y) \geq t f_{n}(x)$.

Let $x_{0} \in X$ be the point with the bounded path $\left\{x_{n}\right\}$ and $y_{0}$ be any point in $X$. Suppose that both cases (i) and (ii) are not true. Then $\left\{\bar{d}\left(x_{n}, y_{n}\right)\right\}$ is bounded. Hence $\lim _{n \rightarrow \infty} \bar{d}\left(x_{n}, y_{n}\right)=0$ by Theorem 2.2. Using Lemma 3.1, we have $\lim _{n \rightarrow \infty}\left\|x_{n}-y_{n}\right\|=0$.

Lemma 3.3. Let $\phi_{n}:(0,1) \rightarrow(0,1), n \geq 1$, be a sequence of lower semicontinuous functions. If $\liminf _{n \rightarrow \infty} \phi_{n}(t)>t$ for all $t \in(0,1)$, then for each $[a, b] \subset(0,1)$, $\liminf \operatorname{in}_{n \rightarrow \infty} \min _{t \in[a, b]} \frac{\phi_{n}(t)}{t}>1$.

Proof. Assume that $\liminf \inf _{n \rightarrow \infty} \min _{t \in[a, b]} \frac{\phi_{n}(t)}{t} \leq 1$ under the given conditions. Then for a given $\epsilon>0$, there exists a subsequence of natural numbers $\left\{n_{k}\right\}$ such that $\min _{t \in[a, b]} \frac{\phi_{n_{k}}(t)}{t}<1+\epsilon$. Hence for each $n_{k}$, we can find $t_{k} \in[a, b]$ which satisfies $\phi_{n_{k}}\left(t_{k}\right)<(1+\epsilon) t_{k}$. There exists a subsequence of $\left\{t_{k}\right\}$, which is still denoted by $\left\{t_{k}\right\}$ for simplicity, such that $\lim _{k \rightarrow \infty} t_{k}=t_{0} \in[a, b]$. By the lower semicontinuity of $\phi_{n_{k}}$, we have $\phi_{n_{k}}\left(t_{0}\right)<(1+\epsilon) t_{0}$ for each $n_{k}$. Since $\epsilon>0$ can be arbitrary, $\phi_{n_{k}}\left(t_{0}\right) \leq t_{0}$, which contradicts that $\liminf _{n \rightarrow \infty} \phi_{n}(t)>t$ for all $t \in(0,1)$.

In the following corollary, we will apply Theorem 3.2 and Lemma 3.3 to a sequence of operators which are related to the ascending operators in $[6]$.

Corollary 3.4. Let $f_{n}: C \rightarrow C, n \geq 1$, be a sequence of monotone operators, and $\phi_{n}:(0,1) \rightarrow(0,1), n \geq 1$, be a sequence of lower semicontinuous functions and $\phi_{n}(t) \geq t$. Suppose that $\liminf _{n \rightarrow \infty} \phi_{n}(t)>t$ for all $t \in(0,1)$, and

$$
y \geq t x \quad \text { implies } \quad f_{n}(y) \geq \phi_{n}(t) f_{n}(x)
$$

for all $t \in(0,1), x, y \in C$. If there exists an $x_{0} \in C$ such that $\sup \left\{\left\|x_{n}\right\|\right\}<\infty$, where $x_{n}=f_{n}\left(x_{n-1}\right), n=1,2, \cdots$, then for any $y_{0} \in C$ and $y_{n}=f_{n}\left(y_{n-1}\right)$, we have the following trichotomy. Either

(i) $\left\{M\left(x_{n} / y_{n}\right)\right\}$ is unbounded; or

(ii) $\left\{M\left(y_{n} / x_{n}\right)\right\}$ is unbounded; or

(iii) $\lim _{n \rightarrow \infty}\left\|x_{n}-y_{n}\right\|=0$.

Proof. We show that condition (10) implies condition (8).

For each $n$, since $\phi_{n}(t)$ is lower semicontinuous, $\log _{t} \phi_{n}(t)$ is upper semicontinuous on $(0,1)$. Hence for each $[a, b] \subset(0,1), \log _{t} \phi_{n}(t)$ attains its maximum $\alpha_{n}(a, b)$ on $[a, b]$.

We claim that $\limsup _{n \rightarrow \infty} \alpha_{n}(a, b)<1$. For if not, then $\limsup _{n \rightarrow \infty} \alpha_{n}(a, b) \geq 1$, i.e.,

$$
\limsup _{n \rightarrow \infty} \max _{t \in[a, b]} \log _{t} \phi_{n}(t) \geq 1
$$

which implies that

$$
\liminf _{n \rightarrow \infty} \min _{t \in[a, b]} \frac{\phi_{n}(t)}{t} \leq 1
$$

It is impossible due to Lemma 3.3. 
Now, if $y>t x$, where $t \in[a, b]$, then

$$
\begin{aligned}
f_{n}(y) & \geq \phi_{n}(t) f_{n}(x) \\
& =t^{\log _{t} \phi_{n}(t)} f_{n}(x) \\
& \geq t^{\alpha_{n}[a, b]} f_{n}(x) .
\end{aligned}
$$

Hence $f_{n}$ 's satisfy condition (8). When $t \bar{\epsilon}[a, b], \phi_{n}(t) \geq t$ implies that $f_{n}(y) \geq$ $t f_{n}(x)$. An application of Theorem 3.2 concludes the proof.

Corollary 3.5. Let $f_{n}: C \rightarrow C, n \geq 1$, be a sequence of monotone operators. Suppose for each $\left[l_{1}, l_{2}\right] \subset(0,1)$, there exists $\alpha\left(l_{1} l_{2}\right)<1$ such that

$$
y \geq t x \quad \text { implies } \quad f_{n}(y) \geq t^{\alpha\left(l_{1} l_{2}\right)} f_{n}(x)
$$

for all $n \geq 1, t \in\left[l_{1}, l_{2}\right]$, and $x, y \in C$. If there exists an $x_{0} \in C$ such that $\sup \left\{\left\|x_{n}\right\|\right\}<\infty$, where $x_{n}=f_{n}\left(x_{n-1}\right), n=1,2, \cdots$, then for any $y_{0}, z_{0} \in C$, and $y_{n}=f_{n}\left(y_{n-1}\right), z_{n}=f_{n}\left(z_{n-1}\right)$, we have $\lim _{n \rightarrow \infty}\left\|y_{n}-z_{n}\right\|=0$.

Proof. Condition (11) and the argument leading to (9) tell us that $\bar{d}\left(x_{n}, y_{n}\right) \leq$ $\bar{d}\left(x_{1}, y_{1}\right)$, i.e., $\left\{\bar{d}\left(x_{n}, y_{n}\right)\right\}$ is bounded. Therefore, both $\left\{M\left(x_{n} / y_{n}\right)\right\}$ and $\left\{M\left(y_{n} / x_{n}\right)\right\}$ are bounded. By Theorem 3.2, $\lim _{n \rightarrow \infty}\left\|x_{n}-y_{n}\right\|=0$. Similarly, $\lim _{n \rightarrow \infty}\left\|x_{n}-z_{n}\right\|=0$. It follows that $\lim _{n \rightarrow \infty}\left\|y_{n}-z_{n}\right\|=0$ due to $\left\|y_{n}-z_{n}\right\| \leq\left\|x_{n}-y_{n}\right\|+\left\|x_{n}-z_{n}\right\|$.

Theorem 3.6. Let $f_{n}: C \rightarrow C, n \geq 1$, be a sequence of operators. Suppose that there exists $\alpha_{n}>0$ with $\lim \sup _{n \rightarrow \infty} \alpha_{n}<1$ such that

$$
y \geq t x \quad \text { implies } \quad f_{n}(y) \geq t^{\alpha_{n}} f_{n}(x)
$$

for all $t \in(0,1]$ and $x, y \in C$. If there exists an $x_{0} \in C$ such that $\sup \left\{\left\|x_{n}\right\|\right\}<\infty$, where $x_{n}=f_{n}\left(x_{n-1}\right), n=1,2, \cdots$, then for any $y_{0}, z_{0} \in C$, and $y_{n}=f_{n}\left(y_{n-1}\right)$, $z_{n}=f_{n}\left(z_{n-1}\right)$, we have $\lim _{n \rightarrow \infty}\left\|y_{n}-z_{n}\right\|=0$.

Proof. Note that (12) implies that $f_{n}$ is monotone (put $\mathrm{t}=1$ ). By a proof similar to the proof of Theorem 3.2 but using Theorem 2.5 instead of Theorem 2,2, we have $\lim _{n \rightarrow \infty}\left\|x_{n}-y_{n}\right\|=0$. Similarly, $\lim _{n \rightarrow \infty}\left\|x_{n}-z_{n}\right\|=0$. It follows that $\lim _{n \rightarrow \infty}\left\|y_{n}-z_{n}\right\|=0$ by the triangle inequality.

\section{WEAK ERGODIC THEOREMS}

The absolute magnitudes of the inhomogeneous iterates may approach infinity. To get the results in Section 3, we imposed the boundedness condition on the path of the sequence of operators at at least one point. Another way to control the growth of the magnitude is to consider the rescaled operators. For $f: P \rightarrow P$ with $f(x) \neq 0$, the rescaled operator to $f$ is defined by $T x=\frac{f(x)}{\|f(x)\|}$. The Hilbert metric is a convenient tool to handle the rescaled operators.

Let $x, y \in C$, where $C$ is a component, and

$$
M(x / y)=\inf \{\lambda \geq 0: x \leq \lambda y\} \text { and } m(x / y)=\sup \{\mu \geq 0: \mu y \leq x\} .
$$

The Hilbert metric is defined by

$$
d(x, y)=\ln \frac{M(x / y)}{m(x / y)}
$$


which is a pseudo-metric since $d(x, y)=0$ if and only if $y=\lambda x$ for some $\lambda>0$. We have the following inequality (Nussbaum [15, (1.20a)])

$$
\|x-y\| \leq M e^{d(x, y)-1} \quad \text { for }\|x\|=\|y\|=1,
$$

where $M$ is a constant.

We denote $S=\{x \in \stackrel{\circ}{P}:\|x\|=1\}$. Then $(S, d)$ is a complete metric space (see Guo and Lakshimikantham [5, Theorem 1.5.2]).

Theorem 4.1. Let $f_{n}: S \rightarrow S, n \geq 1$, be a sequence of operators. Suppose for each $l \in(0,1]$, there exists $\alpha_{n}(l)>0$ with $\limsup _{n \rightarrow \infty} \alpha_{n}(l)<1$ such that

$$
y \geq t x \quad \text { implies } \quad f_{n}(y) \geq t^{\alpha_{n}(l)} f_{n}(x) \quad \text { if } t \in[l, 1]
$$

and $f_{n}(y) \geq t f_{n}(x)$, otherwise, where $x, y \in S$. Then for any $x_{0}, y_{0} \in S$ and $x_{n}=$ $T_{n}\left(y_{n-1}\right), y_{n}=T_{n}\left(y_{n-1}\right)$, where $T_{n}$ is the rescaled operator to $f_{n}$ and $n=1,2, \cdots$, we have the following trichotomy. Either

(i) $\left\{M\left(x_{n} / y_{n}\right)\right\}$ is unbounded; or

(ii) $\left\{M\left(y_{n} / x_{n}\right)\right\}$ is unbounded; or

(iii) $\lim _{n \rightarrow \infty}\left\|x_{n}-y_{n}\right\|=0$.

Proof. First note that (14) implies that $f_{n}$ is monotone (put $t=1$ ). Let $x, y \in S$ with $d(x, y) \in[a, b] \subset(0, \infty)$. Then

$$
e^{a} \leq \frac{M(x / y)}{m(x / y)} \leq e^{b}
$$

Note that $\|x\|=\|y\|=1$ implies $m(x / y) \leq 1 \leq M(x / y)$. It follows from (15) that

$$
1 \leq M(x / y) \leq e^{b} \text { and } e^{-b} \leq m(x, y) \leq 1 .
$$

Using (14),

$$
f_{n}(y) \geq\left[\frac{1}{M(x / y)}\right]^{\alpha_{n}\left(e^{-b}\right)} f_{n}(x)
$$

and

$$
f_{n}(x) \geq m(x / y)^{\alpha_{n}\left(e^{-b}\right)} f_{n}(y),
$$

where $\alpha_{n}\left(e^{-b}\right)>0$. Therefore,

$$
M\left(f_{n}(x) / f_{n}(y)\right) \leq M(x / y)^{\alpha_{n}\left(e^{-b}\right)}
$$

and

$$
m\left(f_{n}(x) / f_{n}(y)\right) \geq m(x / y)^{\alpha_{n}\left(e^{-b}\right)} .
$$

Now

$$
\begin{aligned}
d\left(f_{n}(x), f_{n}(y)\right) & =\ln \frac{M\left(f_{n}(x) / f_{n}(y)\right)}{m\left(f_{n}(x) / f_{n}(y)\right)} \\
& \leq \ln \frac{M(x / y)^{\alpha_{n}\left(e^{-b}\right)}}{m(x / y)^{\alpha_{n}\left(e^{-b}\right)}} \\
& =\alpha_{n}\left(e^{-b}\right) \ln \frac{M(x / y)}{m(x / y)} \\
& =\alpha_{n}\left(e^{-b}\right) d(x, y) .
\end{aligned}
$$

Hence,

$$
d\left(T_{n}(x), T_{n}(x)\right) \leq \alpha_{n}\left(e^{-b}\right) d(x, y) .
$$


Let $L(a, b)=\lim \sup _{n \rightarrow \infty} \alpha_{n}\left(e^{-b}\right)<1$. Then for any given $\epsilon>0$, there exists $N=N(\epsilon, a, b)$ such that

$$
\alpha_{n}\left(e^{-b}\right)<L(a, b)+\frac{\epsilon}{b-a}
$$

for all $n>N$. Thus

$$
d\left(T_{n}(x), T_{n}(y)\right) \leq L(a, b) d(x, y)+\epsilon
$$

for all $n>N$.

Suppose that both cases (i) and (ii) are not true. Then $\left\{d\left(x_{n}, y_{n}\right)\right\}$ is bounded by noting that $m\left(x_{n} / y_{n}\right)=\frac{1}{M\left(y_{n} / x_{n}\right)}$. Hence $\lim _{n \rightarrow \infty} d\left(x_{n}, y_{n}\right)=0$ due to Theorem 2.2. It follows from (13) that $\lim _{n \rightarrow \infty}\left\|x_{n}-y_{n}\right\|=0$.

Corollary 4.2. Let $f_{n}: S \rightarrow S, n \geq 1$, be a sequence of operators. Suppose for each $l \in(0,1]$, there exists $\alpha(l)<1$ such that

$$
y \geq t x \quad \text { implies } \quad f_{n}(y) \geq t^{\alpha(l)} f_{n}(x)
$$

for all $n \geq 1, t \in[l, 1]$, and $x, y \in S$. Then for any $x_{0}, y_{0} \in S$, and $x_{n}=T_{n}\left(x_{n-1}\right)$, $y_{n}=T_{n}\left(y_{n-1}\right)$, where $T_{n}$ is the rescaled operator to $f_{n}$ and $n=1,2, \cdots$, we have $\lim _{n \rightarrow \infty}\left\|x_{n}-y_{n}\right\|=0$.

Proof. A similar argument as in the proof of Theorem 4.1 yields

$$
d\left(T_{n}(x), T_{n}(y)\right) \leq L(a, b) d(x, y)
$$

for all $n$, where $x, y \in S$ and $d(x, y) \in[a, b] \subset(0, \infty)$. An application of Corollary 2.3 finishes the proof.

Theorem 4.3. Let $f_{n}: S \rightarrow S, n \geq 1$, be a sequence of operators. Suppose that there exists $\alpha_{n}>0$ with $\lim \sup _{n \rightarrow \infty} \alpha_{n}<1$ such that

$$
y \geq t x \quad \text { implies } \quad f_{n}(y) \geq t^{\alpha_{n}} f_{n}(x)
$$

for all $t \in[0,1]$ and $x, y \in S$. Then for any $x_{0}, y_{0} \in S$, and $x_{n}=T_{n}\left(x_{n-1}\right)$, $y_{n}=T_{n}\left(y_{n-1}\right)$, where $T_{n}$ is the rescaled operator $f_{n}$ and $n=1,2, \cdots$, we have $\lim _{n \rightarrow \infty}\left\|x_{n}-y_{n}\right\|=0$.

Proof. The proof is similar to the proof of Theorem 4.1 but using Theorem 2.5 instead of Theorem 2.2.

\section{An APPLicAtion to the RANKING PROBLEM}

In this section we consider the change of the ranks of $n$ teams of a paired competition game over time, e.g., football, baseball, etc. There are a variety of methods of paired comparisons being used. In [10], Keener proposed a nonlinear scheme to improve the ranking results. We are going to modify his scheme to study the history of ranks of $n$ teams over discrete points of time.

Let $\mathbf{r}(t) \in R^{n}$ be the ranking vector at time $t, t=0,1,2, \cdots$. Its components $r_{j}(t)$ are positive and represent the strength of the $j$ th team at time $t$. Suppose that $R^{n}$ is partially ordered by the cone $R_{+}^{n}=\left\{\mathrm{x} \in R^{n}: x_{j} \geq 0,1 \leq j \leq n\right\}$ and the norm $\|\mathbf{x}\|=\max _{1 \leq j \leq n}\left|x_{j}\right|$. We propose to calculate the strengths of the $n$ teams at time $t+1$ from the strengths of the $n$ teams at time $t$ and the outcomes of the games at time $t$ as follows:

$$
r_{i}(t+1)=\frac{1}{n_{i}(t)} \sum_{j=1}^{n} f\left(e_{i j}(t) r_{j}(t)\right),
$$


where $i=1,2, \cdots, n, e_{i j}(t) \geq 0$ is a number that is determined from the outcome of the game between team $i$ and $j$ at time $t, n_{i}(t)$ is the number of games played by team $i$ at time $t, f:[0, \infty) \rightarrow[0,1]$ is continuous and strictly increasing, $\lim _{x \rightarrow \infty} f(x)=1$ and

$$
f(c x)>c f(x) \text { for all } c \in[0,1) .
$$

There are different ways to assign the values to $e_{i j}(t)$ 's. For example, at a particular time $t$, we may let $e_{i j}(t)=1$, if team $i$ beat team $j, e_{i j}(t)=\frac{1}{2}$, if team $i$ and $j$ tied, and $e_{i j}(t)=0$, otherwise, $\left(e_{i i}(t)=0\right)$.

From the above formulas, one can see that $\mathbf{r}(t+1)$ is the rankings of $n$ teams before the games at time $t+1$ and can help to predict the outcomes of the games at time $t+1$.

Lemma 5.1. Let $B$ be a compact set in $[0, \infty)$ and $f$ be defined as above. Then for each $\left[l_{1}, l_{2}\right] \subset(0,1)$, there exists $\alpha\left(l_{1}, l_{2}\right) \in(0,1)$ such that

$$
f(c x) \geq c^{\alpha\left(l_{1}, l_{2}\right)} f(x)
$$

for all $c \in\left[l_{1}, l_{2}\right]$ and $x \in B$.

Proof. Let $\left[l_{1}, l_{2}\right] \subset(0,1)$ be given. $\left[l_{1}, l_{2}\right] \times B$ is compact with Cartesian product topology. Let

$$
m\left(l_{1}, l_{2}\right)=\min \left\{\frac{f(c x)}{c f(x)}:(c, x) \in\left[l_{1}, l_{2}\right] \times B\right\} .
$$

It is well defined since $f(0)>0$ (put $c=0$ in (19)) and $f$ is strictly increasing. Furthermore, $m\left(l_{1}, l_{2}\right)>1$ since $f(c x)>c f(x)$. Let $\alpha\left(l_{1}, l_{2}\right)=1+\log _{l_{1}} m\left(l_{1}, l_{2}\right)$ and we claim that $\alpha\left(l_{1}, l_{2}\right) \in(0,1)$. First $\log _{l_{1}} m\left(l_{1}, l_{2}\right)<0$ implies that $\alpha\left(l_{1}, l_{2}\right)<1$. Second, (20) implies that $m\left(l_{1}, l_{2}\right) c f(x) \leq f(c x)<f(x)$, so that $m\left(l_{1}, l_{2}\right) c<1$. This in turn implies $m\left(l_{1}, l_{2}\right) l_{1}<1$, hence $\log _{l_{1}} m\left(l_{1}, l_{2}\right)+1>0$, i.e., $\alpha\left(l_{1}, l_{2}\right)>0$. The claim is proved.

Using (20), we have

$$
\begin{aligned}
f(c x) & \geq m\left(l_{1}, l_{2}\right) c f(x) \\
& =l_{1}^{\alpha\left(l_{1}, l_{2}\right)-1} c f(x) \\
& \geq c^{\alpha\left(l_{1}, l_{2}\right)-1} c f(x) \\
& =c^{\alpha\left(l_{1}, l_{2}\right)} f(x),
\end{aligned}
$$

where $c \in\left[l_{1}, l_{2}\right]$.

Now we have the following theorem.

Theorem 5.2. Let

$$
\mathbf{r}(t+1)=F(t)(\mathbf{r}(t)),
$$

where $F(t)$ is defined by (18). Suppose that there exist two positive constants $M$ and $m, M>m$, such that

$$
M \geq e_{i j}(t) \geq m \quad \text { for all } i, j \text { and } t .
$$

If $\mathbf{x}_{\mathbf{0}}$ and $\mathbf{y}_{\mathbf{0}}$ are any two initial ranking vectors, and $\mathbf{x}_{\mathbf{t}}=T(t) \mathbf{x}_{\mathbf{t}-\mathbf{1}}, \mathbf{y}_{\mathbf{t}}=T(t) \mathbf{y}_{\mathbf{t}-\mathbf{1}}$, where $t=1,2, \cdots$. Then $\lim _{t \rightarrow \infty}\left\|\mathbf{x}_{\mathbf{t}}-\mathbf{y}_{\mathbf{t}}\right\|=0$. 
Proof. This theorem is proved in several steps.

(i) Let $\mathbf{e} \in R^{n}$ be the element with all its components equal to 1 . Then for all $\mathbf{r} \in R_{+}^{n}$, (18) implies

$$
f(0) \mathbf{e}<F(t) \mathbf{r}<n \mathbf{e}
$$

It follows that $\sup \left\{\left\|x_{t}\right\|\right\} \leq n$, and without loss of generality, we can assume that $F(t):[f(0) \mathbf{e}, n \mathbf{e}] \rightarrow[f(0) \mathbf{e}, n \mathbf{e}]$.

(ii) Since only the iterates of $f$ are concerned, (21) and (22) imply that we can assume

$$
M n \geq e_{i j}(t) r_{j} \geq m f(0) .
$$

(iii) For each $\left[l_{1}, l_{2}\right] \subset(0,1)$ and $c \in\left[l_{1}, l_{2}\right]$, in view of (23), we can apply Lemma 5.1 and have

$$
\begin{aligned}
F(t)(c \mathbf{r}) & =\left(\frac{1}{n_{i}(t)} \sum_{j=1}^{n} f\left(e_{i j}(t) c r_{j}\right)\right)_{i=1, \ldots, n} \\
& \geq\left(\frac{1}{n_{i}(t)} \sum_{j=1}^{n} c^{\alpha\left(l_{1}, l_{2}\right)} f\left(e_{i j}(t) r_{j}\right)\right)_{i=1, \ldots, n} \\
& =c^{\alpha\left(l_{1}, l_{2}\right)}\left(\frac{1}{n_{i}(t)} \sum_{j=1}^{n} f\left(e_{i j}(t) r_{j}\right)\right)_{i=1, \ldots, n} \\
& =c^{\alpha\left(l_{1}, l_{2}\right)} F(t)(\mathbf{r}) .
\end{aligned}
$$

(iv) Combining (i)-(iii), we have $\lim _{t \rightarrow \infty}\left\|\mathbf{x}_{\mathbf{t}}-\mathbf{y}_{\mathbf{t}}\right\|=0$ by Corollary 3.5 .

This theorem deals with the long term behavior of the nonlinear scheme proposed in [10], and tells us that using our modified scheme, the rankings of the teams will solely depend on the outcome of the games in the long run, no matter what the initial rankings are. It suggests us that the ranking error or inaccuracy of a particular year can be corrected over time under our scheme.

\section{ACKNOWLEDGMent}

The author is very grateful to the referee for many valuable comments and suggestions.

\section{REFERENCES}

[1] D. W. Boyd and J. S. W. Wong, On nonlinear contractions, Proc. Amer. Math. Soc. 20(1969), 458-464. MR 39:916

[2] T. A. Burton, Integral equations, implicit functions and fixed points, Proc. Amer. Math. Soc. 124(1996), 2383-2390. MR 96j:45001

[3] Y.-Z. Chen, Thompson's metric and mixed monotone operators, J. Math. Anal. Appl. 117(1993), 31-37. MR 94d:47055

[4] Y.-Z. Chen, Inhomogeneous iterates of contraction mappings and nonlinear ergodic theorems, Nonlinear Analysis 39(2000), 1-10. CMP 2000:03

[5] D. Guo and V. Lakshimikantham, Nonlinear Problems in Abstract Cones, Academic Press, New York, 1988. MR 89k:47084

[6] T. Fujimoto and U. Krause, Asymptotic properties for inhomogeneous iterations of nonlinear operators, SIAM J. Math. Anal. 19(1988), 841-853. MR 90b:47119

[7] T. Fujimoto and U. Krause, Stable inhomogeneous iterations of nonlinear positive operators on Banach spaces, SIAM J. Math. Anal. 25(1994), 1195-1202. [MR 95m:47104]

[8] H. Inaba, Weak ergodicity of population evolution processes, Math. Biosc. 96(1989), 195-219. MR 91b:92028 
[9] J. R. Jachymski, An extension of A. Ostrowski's Theorem on the round-off stability of iterations, Aequ. Math. 53(1997), 242-253. MR 98d:47122

[10] J. P. Keener, The Perron-Frobenius Theorem and the ranking of football teams, SIAM Review 35(1993), 80-93. MR 94a:15012

[11] M. A. Krasnosel'skiǐ and P. P. Zabreǐko, Geometrical Methods of Nonlinear Analysis, Springer-Verlag, Berlin, 1984. MR 85b:47057

[12] U. Krause, Path stability of prices in a nonlinear Leontief model, Ann. Oper. Res. 37(1992), 141-148. CMP 93:01

[13] U. Krause, Positive nonlinear systems: some results and applications, Proceedings of the First World Congress of Nonlinear Analysts 1992, W. de Gruyter, Berlin, 1996. CMP 96:12

[14] R. D. Nussbaum, Iterated nonlinear maps and Hilbert's projective metric, Mem. Amer. Math. Soc. Vol.75, No.391 (Sept. 1988). MR 89m:47046

[15] R. D. Nussbaum, Some nonlinear weak ergodic theorems, SIAM. J. Math. Anal. 21(1990), pp. 436-460. MR 90m:47081

[16] E. Seneta, Non-negative Matrices and Markov Chains, 2nd ed., Springer-Verlag, Berlin, 1980; 1st ed., Non-negative Matrice, G. Allen and Unwin, London, 1973. MR 85i:60058

[17] A. C. Thompson, On certain contraction mappings in a partially ordered vector space, Proc. Amer. Math. Soc., 14(1963), 438-443. MR 26:6727

Division of Natural Sciences, University of Pittsburgh at Bradford, Bradford, Pennsylvania 16701

E-mail address: yong@imap.pitt.edu 